Classification

Physics Abstracts

36.20E - 61.12E - 68.45

\title{
About the sensitivity of the small angle neutron scattering technique in the determination of a polymer interfacial density profile
}

\author{
P. Auroy and L. Auvray \\ Laboratoire Léon Brillouin, CEA-CNRS, CEN Saclay, 91191 Gif-sur-Yvette Cedex, France
}

(Received 3 August 1992, accepted in final form 2 November 1992)

\begin{abstract}
We have measured the small angle neutron scattering intensity for two samples consisting of deuterated poly(styrene) chains, grafted onto silica and immersed in a good solvent - so-called polymer brushes. Two different methods have been used : contrast matching and contrast variation. The data have been analysed in terms of an interfacial density profile. Amongst the different forms tested, only the parabolic one has been found to agree satisfactorily with the data. Moreover, the technique is sufficiently sensitive to show that an exponential tail and eventually an adsorption layer should be added in order to obtain a « realistic » interfacial density profile.
\end{abstract}

\section{Introduction.}

For more than ten years, numerous theoretical papers [1] have dealt with grafted polymers. Sophisticated analysis, luminous arguments and also complicated calculations have been proposed to describe the interfaces formed by these grafted chains. The degree of refinement of these theories is at a very high level. By comparison, the current experimental methods are very crude. But this gap has stimulated the experimentalists and much effort has been put in, especially in two directions: chemistry and measurement. New molecules (disymmetrical diblock copolymers, for instance) have been synthesized, special care has been taken to control the interaction between the surface and the macromolecules... A good design in the experimental system is the first requirement for a meaningful physical measurement on these grafted chains.

But this prerequesite condition is not completely sufficient. In this paper, we will focus on the second sine qua non: the use of a sensitive experimental technique with a high level of resolution. Roughly, one can distinguish 3 methods that are now able to give a picture of the density profile of grafted chains : the surface-force apparatus, small-angle neutron scattering (SANS) technique and neutron reflectivity. The first method has allowed useful information [2] to be gained about the interactions between two surfaces bearing adsorbed or grafted 
chains. To date, this achievement has not been equalled. However, the density profile is only indirectly derived from the law of the force versus the distance between the two plates. Furthermore, it appears that the interpretation of the experimental results, in terms of interfacial density profile, depends on the theoretical model that is introduced for fitting the data. For instance, in the case of brushes (grafted chains with a high grafting density), the two traditional predictions for the density profile : step [3] and parabola [4] have been found to agree very well with the experiments.

The latter two techniques, SANS and neutron reflectivity, differ completely from the former and they can be considered as having certain similarities. The use of the SANS technique, in the context of polymers at interfaces, has been developed for more than ten years and the performances of these experiments have been constantly improved. There was a debate concerning the interpretation of the data obtained with this SANS technique : the problem is that the raw results are expressed in terms of a scattering intensity $I(q)$ versus the scattering vector $q$. How should one convert the data (in the reciprocal space) to an interfacial density profile $\phi(z)$ ? ( $z$ is the coordinate normal to the surface). The Bristol group (Cosgrove, Crowley and Vincent) has defined [5] and used a strategy that requires a strong mathematical formalism but that indeed allows one to determine (in principle, uniquely) $\phi(z)$. Our group has proposed a different approach [6], that attaches great importance to the direct analysis of the data in the reciprocal space. In general, this does not allow $\phi(z)$ to be precisely determined, but it gives the main features of the density profile. In particular, it is very sensitive to some singularities of $\phi(z)$ like a power law decrease $\left(z^{-\alpha}\right)$ or a discontinuity. Moreover, the determination of $\phi(z)$ is not always feasible with this SANS technique unless two complementary experiments are done on the same sample. Namely, a contrast matching experiment and a contrast variation. (This will be recalled in the first part of this paper.) These two are absolutely required if there are some concentration fluctuations within the interface. (This occurs especially when the chains are immersed in a good solvent.) We now believe that the two approaches are complementary and in particular, it is necessary to go beyond our former « qualitative » description of $\phi(z)$ if one wants to discuss the results of the most recent theories.

The reflectivity technique has been used much more recently for studying polymers at interfaces [7]. It has been claimed that it is more appropriate than the SANS for determining $\phi(z)$. Indeed, there is no contribution to the scattering due to the concentration fluctuations. Therefore, with an adequate formalism, it is possible, in principle, to directly interpret the data in terms of an average density profile. However, this is an optimistic view and it appears that many artifacts can be introduced into the whole experimental procedure (roughness of the substrate - more or less known -, shift of the critical angle, strange isotopic effects, insufficient resolution...). As a result, the determination of $\phi(z)$ is not so ambiguous and special care has to be taken with this technique in order to draw definitive conclusions.

In this paper, we aim to present the most recent developments of our SANS technique when it is used for studying polymer interfaces. Two samples (same as in Ref. 8) will be considered : both consist of poly(styrene) brushes. We have already reported [8] the physical picture of these two samples we can obtain with our SANS technique. In this paper, we aim to discuss in more detail the accuracy of this investigation. Our purpose is not to describe a general way for inverting any kind of scattering intensity spectrum but to show for a given sample (especially well designed) that this technique is very sensitive to a slight difference in the interfacial density profile. Obviously, there are several brute force inversion procedures published in the literature that could be used for deriving $\phi(z)$ directly. But it is beyond the scope of this paper to compare all these mathematical treatments. Our strategy is lighter and somewhat more straightforward. Nevertheless, we do not claim that it is the unique (and the best) way for obtaining $\phi(z)$. 
In the first part, we shall describe briefly the 2 samples investigated in this paper. We will also recall a few basic concepts about the experimental technique and present the broad lines of the data treatment. In the second part, we attempt to fit the data with different shapes for the interfacial density profile (in particular, with $\phi\left(1-(z / h)^{n}\right)$ with $n=1,2$ or 3 ) and we shall compare all these results. In the last section, we discuss the importance and the meaning of some « corrections » which must be added to the preceding forms of $\phi(z)$ in order to improve the quality of the fit with the data. We treat these corrections either as a background (3.1) or as a proximal region (3.2) that would reflect some specific short range interactions between the wall and the polymer.

\section{Experimental section.}

1.1 EXPERIMENTAL SYSTEM. - The two samples are the same as those of reference [8]. They consist of deuterated polystyrene chains $\left(M_{\mathrm{w}}=69000\right.$, polydispersity index : 1.11) grafted onto porous silica. They differ only in their grafting density which is of $9.66 \mathrm{mg} / \mathrm{m}^{2}$ for sample 1 and $5.98 \mathrm{mg} / \mathrm{m}^{2}$ for sample 2 (See Tab. I). We have investigated the structure of these interfacial layers in dichloromethane which is a good solvent for polystyrene. In such a solvent, both samples can be considered as model brushes since their average distance between grafting sites $D$ is much smaller than the radius of gyration of the free chains (that is of the order of $90 \AA$ ).

Table I. - Main experimental characteristics of the two samples of this study. Bold figures: values obtained independently of any model for $\phi(z)$ (otherwise, the parabolic model has been used).

\begin{tabular}{|c|c|c|c|c|}
\hline & & $\begin{array}{c}\gamma_{\mathrm{q} \rightarrow 0} \\
(\AA)\end{array}$ & $\begin{array}{c}\mathrm{D} \\
(\AA)\end{array}$ & $\phi_{\mathrm{s}}^{\text {asymp }}$ \\
\hline \multirow{2}{*}{ Sample 1 } & from I $_{\mathrm{pp}}$ & $\mathbf{8 4}$ & $\mathbf{3 4}$ & $\mathbf{0 . 1 9}$ \\
\cline { 2 - 5 } & from I $\mathrm{pg}$ & 74 & 37 & $\mathbf{0 . 2 3}$ \\
\hline \multirow{2}{*}{ Sample 2 } & from I & $\mathbf{5 2}$ & $\mathbf{4 4}$ & $\mathbf{0 . 1 3}$ \\
\cline { 2 - 5 } & from I & 44 & 48 & $\mathbf{0 . 1 5}$ \\
\hline
\end{tabular}

The deuterated chains have been synthetized by anionic polymerization, in toluene, using sec-butyl-lithium as initiator and terminated by $\mathrm{Cl}_{2} \mathrm{Si}\left(\mathrm{CH}_{3}\right)$. They have been then precipitated by dry methanol in order to convert the chlorosilyl end-group into a methoxy-silyl group. They have then been grafted in solution in carefully dried benzene, at relatively high concentration (60\% for sample 1 and $30 \%$ for sample 2). After the grafting (at temperature of $90^{\circ} \mathrm{C}$, for 24 hours), the silica has been rinsed by pure dichloromethane several times in order to eliminate all the chains which have not reacted. Non-reactive chains have also been synthesized by the same method. They differ only in their termination which is a trimethylsilyl end-group. These chains have been used in order to check that the adsorption is negligible.

1.2 EXPERIMENTAL TECHNIQUE. - All the details about our SANS technique have already been reported [6]. We remind the reader that we measure the scattering intensity $I(q)$ (in $\mathrm{cm}^{-1}$ ) as a function of the scattering vector $q\left(\right.$ in $\AA^{-1}$ ). This scattering intensity has 
been put at the absolute scale by normalizing all the spectra with the incoherent scattering of water. For our three-component system :

$$
I(q)=I_{\mathrm{gg}}(q)+I_{\mathrm{pp}}(q)+I_{\mathrm{pg}}(q)
$$

where the $I_{\mathrm{ij}}$ are the 3 partial structure factors ( $\mathrm{g}$ refers to the silica grains and $\mathrm{p}$ to the polymer). If we adjust the scattering length density of the solvent equal to that of the silica, then both $I_{\mathrm{gg}}$ and $I_{\mathrm{pg}}$ vanish in equation (1). This corresponds to a contrast matching experiment. The neutron refractive index of the solvent is adjusted by using different $H-D$ isotopic mixtures. We call $x_{\mathrm{D}}$ the volume fraction of the perdeuterated component in these mixtures. For our system, the contrast matching is achieved by using dichloromethane with $x_{\mathrm{D}}=0.90$. Under this condition, equation (1) reduces to :

$$
I(q)=\bar{I}_{\mathrm{pp}}(q)+\tilde{I}_{\mathrm{pp}}(q) .
$$

The first term corresponds to the scattering of the average density profile and the second one to the contribution of the concentration fluctuations. It can be shown [9] that, for a flat substrate :

$$
\bar{I}_{\mathrm{pp}}(q)=2 \pi(S / V)\left(n_{\mathrm{p}}-n_{\mathrm{s}}\right)^{2} q^{-2}\left|\int_{0}^{+\infty} \phi(z) \mathrm{e}^{i q z} \mathrm{~d} z\right|^{2}
$$

where $S / V$ is the specific surface area of the substrate and $n_{\mathrm{p}}\left(n_{\mathrm{s}}\right)$ is the scattering length density of the polymer (solvent). Expression (3) is valid for any contrast condition. For our system, $n_{\mathrm{p}}=6.543 \times 10^{10} \mathrm{~cm}^{-2}$ and $n_{\mathrm{s}}=3.484 \times 10^{10} \mathrm{~cm}^{-2}$ (under the contrast matching condition). It can be noticed that the use of deuterated polystyrene is much more appropriate for a contrast matching experiment than ordinary polymer. Indeed, assuming that the grafting density is kept fixed, the scattering intensity is 2.2 times greater just because of the higher contrast between the polymer and the solvent.

A Taylor expansion of (3) leads to :

$$
\bar{I}_{\mathrm{pp}}(q)=2 \pi(S / V)\left(n_{\mathrm{p}}-n_{\mathrm{s}}\right)^{2} q^{-2} \gamma^{2}\left(1-\frac{q^{2} h^{2}}{\alpha}\right)
$$

where $\gamma=\int \phi(z) \mathrm{d} z$ is independent of the shape of the density profile in contrast to $\alpha$ which is a numerical constant depending on the shape of $\phi(z)$. Thus, an extrapolation of $q^{2} l(q)$ at $q \approx 0$ (analogous to a Guinier plot) whatever the shape of $\phi(z)$, gives the amount of polymer per unit area $\gamma . \gamma$ is a general invariant and in particular, it does not depend on the solvent quality. As we have already shown [6], the use of a poor solvent for determining $\gamma$ is much more adequate because in such a solvent, the layer is almost fully collapsed. The Guinier regime under this condition is better defined, allowing a precise measurement of $\gamma$. Therefore, $\gamma$ - measured in a poor solvent - is a fundamental parameter, obtained independently of any model. There is also a second basic parameter which is worked out without any assumption for $\phi(z)$, namely the surface fraction occupied by the polymer $\phi_{\mathrm{s}}=\phi(z=0)$. Indeed, it can be shown [6] that, at high $q$ :

$$
\bar{I}_{\mathrm{pp}}(q)=2 \pi(S / V)\left(n_{\mathrm{p}}-n_{\mathrm{s}}\right)^{2} q^{-4} \phi_{\mathrm{s}}^{2}
$$

This typical $q^{-4}$ behavior (Porod's law) is characteristic of any « regular » and finite scattering medium.

We do not know exactly the structure of the $\tilde{I}_{\mathrm{pp}}$ term but we have observed [8] that 
$\tilde{I}_{\mathrm{pp}}(q)$ is proportional to $\frac{1}{1+q^{2} \xi^{2}}$ provided that $q \xi<1$ where $\xi$ is the typical correlation length of the concentration fluctuations. This Lorentzian behavior is similar to the scattering of any semi-dilute polymer solution.

The polymer-solid structure factor $I_{\mathrm{pg}}(q)$ can be derived by performing a contrast variation experiment, as explained in reference [6]. The general expression for $I_{\mathrm{pg}}(q)$ is :

$$
I_{\mathrm{pg}}(q)=-4 \pi\left(n_{\mathrm{p}}-n_{\mathrm{s}}\right)\left(n_{\mathrm{g}}-n_{\mathrm{s}}\right) q^{-3} \int \phi(z) \sin (q z) \mathrm{d} z
$$

$I_{\mathrm{pg}}(q)$ is not sensitive to the concentration fluctuations. It is important to realize that both expressions (3) and (5) follow asymptotically the same general Porod law ( $q^{-4}$ decrease), but the deviations from this behavior (in the intermediate $q$ range) which are characteristic of the shape of the density profile are completely different. Therefore, if a particular form of $\phi(z)$ fits both, it will be seen as a very likely profile.

As for $\bar{I}_{\mathrm{pp}}(q), I_{\mathrm{pg}}$ allows us to determine the fundamental parameter $\phi_{s}$, independently of any model. But instead of (4), one has :

$$
I_{\mathrm{pg}}(q) \approx-4 \pi(S / V)\left(n_{\mathrm{p}}-n_{\mathrm{s}}\right)\left(n_{\mathrm{g}}-n_{\mathrm{s}}\right) q^{-4} \phi_{\mathrm{s}} .
$$

In order to simplify the notation, we introduce the imaginary function

One then has :

$$
G(q)=\int_{0}^{+\infty} \phi(z) \mathrm{e}^{i q z} \mathrm{~d} z
$$

$$
\begin{aligned}
& \bar{I}_{\mathrm{pp}}(q)=2 \pi(S / V)\left(n_{\mathrm{p}}-n_{\mathrm{s}}\right)^{2} q^{-2}|G(q)|^{2} \\
& I_{\mathrm{pg}}(q)=-4 \pi(S / V)\left(n_{\mathrm{p}}-n_{\mathrm{s}}\right)\left(n_{\mathrm{g}}-n_{\mathrm{s}}\right) q^{-3} \operatorname{Im}(G(q))
\end{aligned}
$$

1.3 DATA TREATMENT. - In this paper, we have restricted the choice for $\phi(z)$ to simple forms that allow us to derive analytical expressions for equations (3) and (5). All these different forms contain 4 adjustable parameters. For a given form of $\phi(z)$, these 4 parameters have been determined by a classical procedure : minimization of chi-square. The experimental errors have been estimated assuming a Gaussian statistics for the raw counts. After having determined the best parameters for each given form of $\phi(z)$, we can compare these different forms and select the best one by using three criteria :

i) for each sample, the same $\phi(z)$ should fit the data obtained with the 2 methods : contrast matching and contrast variation (Eqs. (3) and (5)).

ii) $\phi(z)$ should be consistent with the experimental values for $\gamma$ and $\phi_{\mathrm{s}}$ obtained independently of any model.

iii) The best $\phi(z)$ corresponds to the least $x^{2}$.

All these three criteria make the determination of $\phi(z)$ very constraining; this will make the distinction between very close forms of $\phi(z)$ possible.

\section{Test of $z^{n}$ with $n=1,2$ or 3 .}

2.1 GENERAL. - In this section, we determine the parameters that best fit the data for 3 types of $\phi(z): \phi(z)=\phi_{\mathrm{s}}\left(1-\left(\frac{z}{h}\right)^{n}\right)$ with $n=1,2$ or 3 , corrected by an exponential tail that allows the density profile to decay to zero at large distances more gradually than the pure 
polynomial forms do. The meaning of this correction will be discussed in section 3 in the particular case of the parabolic profile. It is assumed that $\phi(z)$ as well as its derivative remains continuous. Therefore the introduction of an exponential tail gives only a single additional parameter, namely the distance at which this exponential starts : $h_{0}$. For instance, in the case of a parabola, one has:

$$
\begin{gathered}
\phi(z)=\phi_{\mathrm{s}}\left(1-\left(\frac{z}{h}\right)^{2}\right) \quad \text { for } \quad z \leqslant h_{0} \\
\phi(z)=\phi_{\mathrm{s}} \frac{h^{2}-h_{0}^{2}}{h^{2}} \exp \left(-2 h_{0} \frac{z-h_{0}}{h^{2}-h_{0}^{2}}\right) \quad \text { for } \quad z \geqslant h_{0} .
\end{gathered}
$$

It is easy to calculate $G(q)$ corresponding to this $\phi(z)$. One has :

$$
G(q)=\phi_{s}\left(1-u^{2}\right)^{2} \frac{h_{0}}{2 u^{2}-i q h_{0}\left(1-u^{2}\right)} \mathrm{e}^{i q h_{0}}
$$

with $u=\frac{h_{0}}{h}$. From $G(q)$, one can derive an analytical expression for $\bar{I}_{\mathrm{pp}}(q)$ and $I_{\mathrm{pg}}(q)$. The leading term in these two expressions corresponds to a $q^{-4}$ decrease. This typical Porod behavior is general for all the regular $\phi(z)$ we will test in this paper. This leads us to use

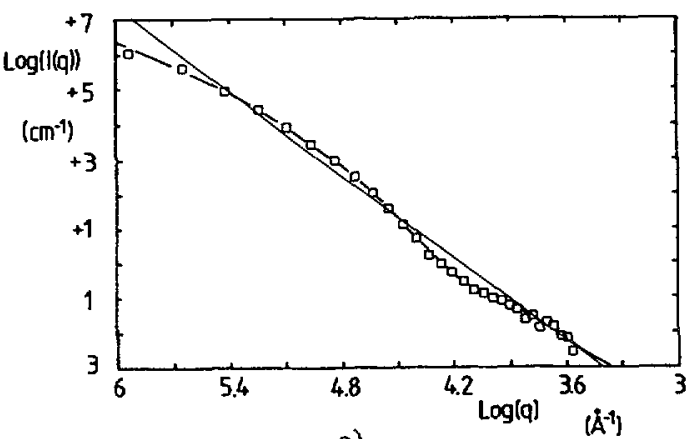

a)

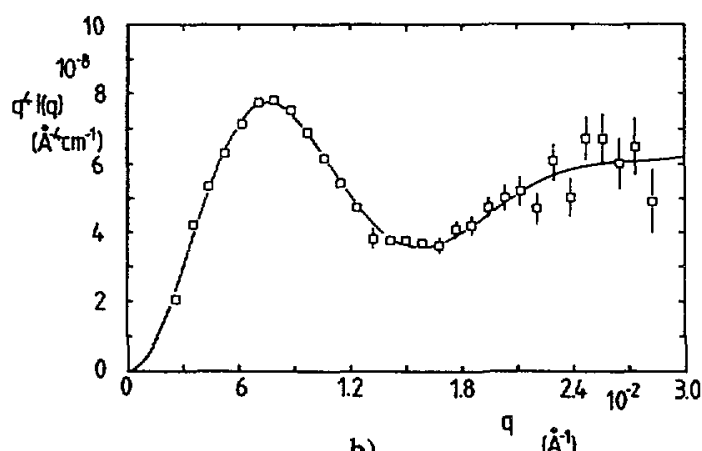

b)

Fig. 1. - Experiment performed at contrast match. a) Plot of the logarithm of the scattering intensity versus the momentum transfer. The straight line has a slope of -4 . The solid curve is the result of the fit with a parabolic profile - see 2.4. b) Same data as for a) but now with the plot of $q^{4} I(q)$ versus $q$.

a $q^{4} I(q)$ versus $q$ plot (instead of $I(q)$ versus $q$ ). A comparison of the different representations is shown in figure 1 , where we have reported the same data (same experimental error bars and same fit) but using these two different plots. With the Log $(I(q))$ versus $\log (q)$ plot, it is not possible to detect any particular feature. The fit seems to be perfect, whereas with the $q^{4} I(q)$ versus $q$ plot, the typical oscillations appear and the comparison between the experimental data and the curve resulting from the fit seems to be meaningful.

$\phi_{s}, h_{0}$ and $h$ are the 3 most important parameters. We have incorporated an additional variable, noted $\gamma_{0}$, whose role is to allow for a «background 》 of the form of $\frac{\gamma_{0}}{q^{2}}$. This « special » parameter and its significance will be discussed in the next part.

As shown in reference [8], the spectra at contrast matching exhibit two distinctive behaviors : at high $q\left(q>3 \times 10^{-2} \AA^{-1}\right)$, the scattering is dominated by the concentration fluctuations and follows a Lorentzian law (cf. Sect. 1.2). For $q<3 \times 10^{-2} \AA^{-1}$, the scattering 
comes mostly from the average density profile. We can therefore consider, in a first approximation, that for $q<3 \times 10^{-2} \AA^{-1}$, the scattering intensity under contrast matching condition reduces only to $\bar{l}_{\mathrm{pp}}(q)$. The contribution of the concentration fluctuations will be seen as a correction to the total scattering intensity and will be discussed in the last part.

2.2 Results. - Tables II, III and IV show the results of the fit with the 3 types of density profile (respectively with $n=1,2$ and 3). Typical curves are also shown in figures 2 and 3 .

We can make a preliminary remark that is general for the 3 forms : both $\phi_{s}$ and $\gamma$ are systematically smaller when they are derived with the contrast variation method than when they are obtained with the contrast matching experiment. We do not know exactly the origin of this discrepancy. We believe that it could be ascribed to uncorrect normalization, that is much more crucial for the contrast variation method because this involves subtraction of spectra obtained at different contrasts. Weak multiple scattering could also be another possible explanation. Again, this would occur especially with the contrast variation method because of the use of isotopic mixtures that contain relatively large volume fraction of hydrogen. Nevertheless, these discrepancies are smaller than $10 \%$ (the overall reproducibility of our experiments) and they do not make the discussion about the shape of the density profile questionable.

Table II. - Values of the different parameters characterizing the best density profile when it is assumed to be linear. See the text for the meaning of these different parameters. $A$ "background" of the form of $\frac{\gamma_{0}}{q^{2}}$ has been used. $\gamma_{0}$ is in $\mathrm{cm}^{-1} \AA^{-2}$

\begin{tabular}{|c|c|c|c|c|}
\hline \multirow{2}{*}{} & \multicolumn{2}{|c|}{ Sample 1 } & \multicolumn{2}{c|}{ Sample 2 } \\
\cline { 2 - 5 } & from I $_{\mathrm{pp}}$ & from I $_{\mathrm{pg}}$ & from I $_{\mathrm{pp}}$ & from I $_{\mathrm{pg}}$ \\
\hline$\phi_{\mathrm{s}}$ & .2594 & .247 & .1848 & .171 \\
\hline $\mathrm{h}_{0}(\mathrm{~A})$ & 574 & 610 & 477 & 535 \\
\hline $\mathrm{h}_{(\mathrm{A})}$ & 595 & 615 & 530 & 536 \\
\hline $\mathrm{u}_{\mathrm{yyyy}}$ & 0.965 & 0.992 & 0.900 & 0.998 \\
\hline$\gamma_{(A)}$ & 77.27 & 75.96 & 49.46 & 45.83 \\
\hline$\gamma_{0}$ & 0 & 0 & 0 & 0 \\
\hline$\chi^{2}$ & 22.64 & 25.60 & 7.711 & 16.21 \\
\hline
\end{tabular}

The first criterion (defined in Sect. 1.3) seems to be satisfied for the 3 types of density profile, though the linear form exhibits great relative variations for $h_{0}$ if we compare the contrast matching and the contrast variation experiments. However, this might not be very significant and criterion 1 appears to be insufficiently selective. On the contrary, the other two criteria lead to the conclusion that the only curve that agrees satisfactorily with the experimental data corresponds to $n=2$. 
Table III. - Same as for table II but the density profile is now assumed to be parabolic.

\begin{tabular}{|c|c|c|c|c|}
\hline \multirow{2}{*}{} & \multicolumn{2}{|c|}{ Sample 1 } & \multicolumn{2}{c|}{ Sample 2 } \\
\cline { 2 - 5 } & from I $_{\mathrm{pp}}$ & from I $_{\mathrm{pg}}$ & from I $_{\mathrm{pp}}$ & from I $_{\mathrm{pg}}$ \\
\hline$\phi_{\mathrm{S}}$ & .2139 & .2000 & .1498 & .1384 \\
\hline $\mathrm{h}_{0}(\mathrm{~A})$ & 475 & 490 & 401 & 419 \\
\hline $\mathrm{h}_{(A)}$ & 566 & 559 & 488 & 474 \\
\hline $\mathrm{u}$ & .839 & .877 & .822 & .884 \\
\hline$\gamma_{(A)}$ & 84.06 & 76.32 & 51.24 & 44.66 \\
\hline$\gamma_{0}$ & $8.010^{-5}$ & $1.210^{-5}$ & $3.410^{-5}$ & $3.410^{-6}$ \\
\hline$\chi^{2}$ & 3.492 & 2.755 & 1.410 & 3.090 \\
\hline
\end{tabular}

2.3 LINEAR FORM. - If we focus in table II which displays the results of the best fit for the linear form, we can notice that the ratio $u=\frac{h_{0}}{h}$ that represents the relative weight of the exponential tail is close to 1 . This indicates that this correction is virtually unecessary. No such foot is needed when we try to fit the data with $\phi_{\mathrm{s}}\left(1-\frac{z}{h}\right)$. By comparison with the other form for $\phi(z)$, this tells us that the curvature of the « exact » density profile should be essentially downwards.

Table IV. - Same as for table II but the density profile is now assumed to be cubic.

\begin{tabular}{|c|c|c|c|c|}
\hline \multirow{2}{*}{} & \multicolumn{2}{|c|}{ Sample I } & \multicolumn{2}{c|}{ Sample 2 } \\
\cline { 2 - 5 } & from I $\mathrm{pp}$ & from I $_{\mathrm{pg}}$ & from I $_{\mathrm{pp}}$ & from I $_{\mathrm{pg}}$ \\
\hline$\phi_{s}$ & .2037 & .185 & .1389 & .1244 \\
\hline$h_{0}(\mathrm{~A})$ & 424 & 431 & 374 & 389 \\
\hline$h_{(A)}$ & 531 & 518 & 459 & 449 \\
\hline$u$ & .798 & .832 & .815 & .866 \\
\hline$\gamma_{(A)}$ & 89.00 & 76.55 & 51.67 & 43.56 \\
\hline$\gamma_{0}$ & $1.1 \times 10^{-4}$ & $2.4 \times 10^{-5}$ & $5.6 \times 10^{-5}$ & $1.5 \times 10^{-5}$ \\
\hline$\chi^{2}$ & 16.42 & 13.30 & 2.085 & 6.773 \\
\hline
\end{tabular}




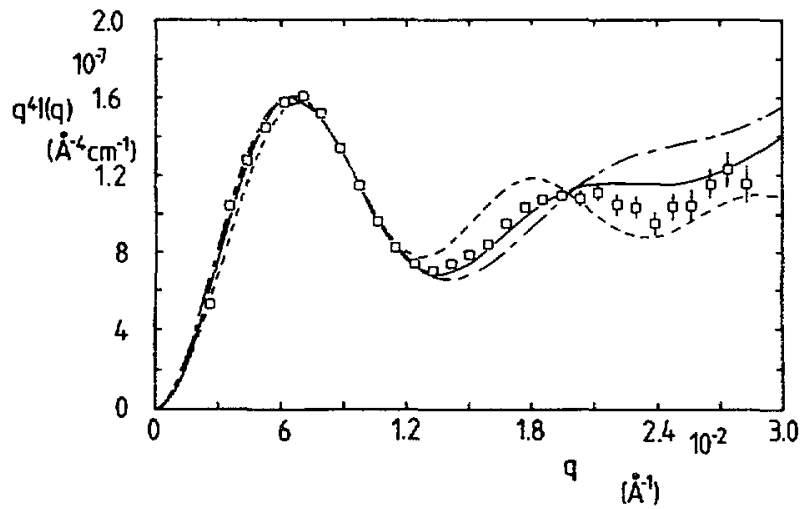

Fig. 2. - Sample 1. Experiment performed at contrast match. Plot of $q^{4} I(q)$ versus $q$. Dashed (solid, dotted-dashed) curve : best fit with a linear (parabolic, cubic) profile.

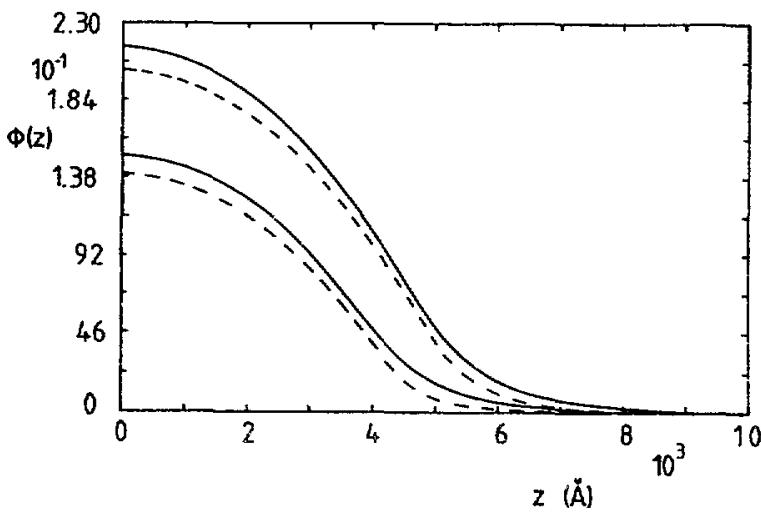

Fig. 3. - Comparison between the polymer density profile obtained from the experiment performed at contrast match (solid curve) and that obtained with the contrast variation method (dashed curve). The two upper curves correspond to sample 1. Parabolic form - see 2.4.

The second remark we can make about this table II is that $\gamma_{0}$ is equal to $0 . \gamma_{0}$ has not been allowed to be negative. As explained in the last part, $\gamma_{0}$ could be interpreted as the signature of a thin adsorption layer (whatever it comes from). The fact that $\gamma_{0}$ is equal to 0 with the linear form indicates that this kind of profile overestimates $\phi(z)$ close to the wall and especially $\phi_{s}$.

Obviously, we could extend the same discussion to the cubic form. In this case we would draw exactly the opposite conclusion; namely, the exponential tail is too important and the surface fraction is underestimated with this cubic form.

2.4 PARABOLIC Form. - The parabolic form agrees rather well with the data and seems to be close to the " exact " picture. The same shape is correct for the two samples, though they have a substantially different grafting density [10].

If we calculate the product $h D^{2 / 3}$ where $D$ is the distance between grafting points for the 4 sets of data of this article, we find something nearly constant (see Tab. V). This is in good agreement with the theoretical prediction [3,4] and with our previous study on grafted polydimethylsiloxane [11]. 
Table V. - Check of the scaling law $h D^{2 / 3}=a^{5 / 3} N . N$ is the polymerization index of the chains. Here, $N=663$. a has the dimension of a length and should be of the same order of magnitude as a molecular size. The values of this table have been calculated using the results of the parabolic model shown in table III.

\begin{tabular}{|c|c|c|c|c|c|}
\hline & & $\begin{array}{c}D \\
(\AA) \\
(\AA ̊)\end{array}$ & $h D^{2 / 3}$ & $\frac{\mathrm{hD}^{2 / 3}}{\mathrm{~N}}$ & $(\stackrel{a}{\grave{A}})$ \\
\hline \multirow{2}{*}{ Sample 1} & from I $_{p p}$ & 34.425 & 5689.62 & 9.24 & 3.80 \\
\hline & from $I_{p g}$ & 36.129 & 6109.12 & 9.92 & 3.96 \\
\hline \multirow{2}{*}{ Sample 2} & from $I_{p p}$ & 44.092 & 6090.64 & 9.89 & 3.95 \\
\hline & from $I_{p g}$ & 47.229 & 6193.24 & 10.05 & 3.99 \\
\hline
\end{tabular}

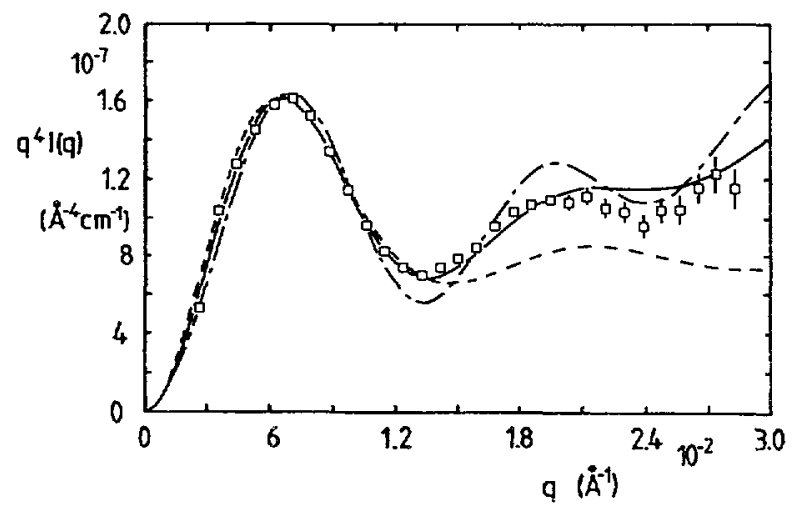

Fig. 4. - Plot of $q^{4} I_{\mathrm{pp}}(q)$ versus $q$. Sample 1. Experiment performed at contrast match. Best fit with the parabolic model corrected by an exponential tail (dashed curve) or by an adsorption layer (dashed-dotted) or by both (solid).

Table VI. - Comparison of the fit with a parabolic model modified with an exponential foot or with an adsorption layer ("background $»$ of the form of $\left.\frac{\gamma_{0}}{q^{2}}\right)$ or with both.

\begin{tabular}{|c|c|c|c|}
\hline & $\begin{array}{c}\text { Exp. tall }+ \\
\text { buckground" }\end{array}$ & Background & Exp. tal \\
\hline$\phi_{s}$ & .2139 & .2039 & .2270 \\
\hline$h_{(\AA)}$ & 566 & 562 & 572 \\
\hline$\gamma_{(\mathcal{A})}$ & 84.06 & 76.39 & 94.50 \\
\hline$h_{0}(\mathcal{A})$ & 475 & $/$ & 438 \\
\hline$\gamma_{0}$ & $8.0 \times 10^{-5}$ & $1.02 \times 10^{-4}$ & $/$ \\
\hline$\chi^{2}$ & 3.492 & 19.27 & 29.49 \\
\hline
\end{tabular}


It is important to estimate the meaning of the two corrections we have added to the "pure " parabolic model, namely $\gamma_{0}$ and the exponential tail. These have been introduced in order to describe two different features of all spectra at large $q: \gamma_{0}$ accounts for the overall vertical shift of the spectra whereas the exponential tail accounts for the (over)damping of the oscillations. This is especially shown in figure 4 where we have reported the comparison between the fits when one of these two corrections is suppressed. However, it is very clear (see Tab. VI) that the two main parameters $\phi_{\mathrm{s}}$ and $h$ remain almost unchanged. The introduction of $\gamma_{0}$ and the exponential tail allows for a more detailed description of the density profile but it does not question the validity of the parabolic shape [12].

About the exponential tail : it would be very interesting to seek whether this foot and its importance depend on the chain length. Indeed, for our sample, the slope of this exponential is equal to $82 \AA$ ( $\pm 8 \AA$ ) close to the radius of gyration of the free polymers in good solvent $(\approx 90 \AA)$. This suggests that the tail would be a correction due to the finite length of the chain, as anticipated by Witten et al. [13].

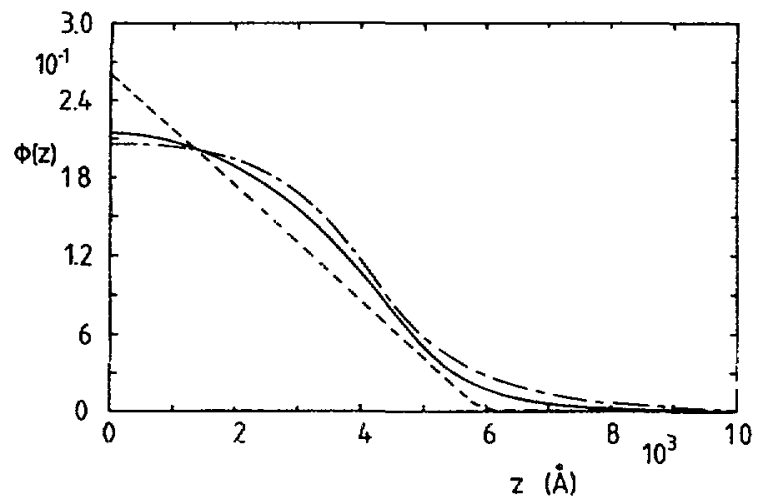

Fig. 5. - Comparison between the 3 types of density profiles. Dashed line : linear. Solid line : parabolic. Dashed-dotted : cubic. All these 3 curves correspond respectively to the best fit with the data obtained with sample 1 by contrast matching.

The most important result of our study is shown in figure 5 where we compare the three types of density profile which correspond respectively to the best fit with the experimental data for the same sample. The three curves are very close; but only the one corresponding to $n=2$ agrees satisfactorily with the experiment. This clearly shows that our method is actually sensitive to the shape of the interfacial density profile and the debate whether $\phi(z)$, in good solvent, is a step or a parabola is now completely settled.

\section{3. $\gamma_{0}$. background or adsorption layer ?}

As we have already explained, we have introduced a special parameter $\gamma_{0}$ that allows us to improve the quality of the fit with the data. The influence of this parameter is significant only at high $q\left(q>10^{-2} \AA^{-1}\right)$. So far, it has been considered as the signature of an (infinitely) thin adsorption layer, leading to an additional scattering intensity of the form of $\frac{\gamma_{0}}{q^{2}}$. This form has been found to agree rather satisfactorily with the experimental data. Nevertheless, it remains questionable and, in particular, it is not consistent if we compare the results of the contrast 
matching experiment with those of the contrast variation method (difference of more than one order of magnitude).

3.1 BACKGROUND. - An alternative explanation for this extra contribution to the scattering intensity could be concentration fluctuations. This would be consistent with the fact that this contribution seems to be much more important for the experiments done under contrast matching condition. Indeed, as explained in reference [9], these concentration fluctuations are detectable only at contrast match $\left(\tilde{I}_{\mathrm{pp}}(q)\right.$ term $)$ and in principle, they are averaged to zero with the contrast variation method. At high $q$, we have already seen that $\tilde{I}_{\mathrm{pp}}(q)$ follows a Lorentzian law. Therefore, if one extrapolates this behavior at very small angles, this yields a constant term [14] that has to be added to the scattering intensity due to the average density profile. This scheme has been tested and the results of the fit are shown in table VII and figure 6.

By comparison with the case of a $\frac{\gamma_{0}}{q^{2}}$ contribution (Tab. III and also Fig. 6), it is clear that this " background », whatever its origin and its form, has no influence on the determination of the interfacial density profile ; the parabolic form agrees with the data best and, moreover, the parameters remain unchanged. Nevertheless, neither the $\frac{\gamma_{0}}{q^{2}}$ nor the $\gamma_{0}$ form provides a full interpretation of the spectra. The origin of this background seems to be complex and it might be the result of various phenomena, including concentration fluctuations and adsorption layer.

3.2 ADSORPTION LAYER. - As we have already explained, the $\frac{\gamma_{0}}{q^{2}}$ contribution we have introduced to improve the quality of the fit can be interpreted as an (infinitely) thin adsorption layer.

Table VII. - Same as for table III but now the "background" is of the form of $\gamma_{0}$ instead of $\frac{\gamma_{0}}{q^{2}}$. Therefore, $\gamma_{0}$ is here in $\mathrm{cm}^{-1}$

\begin{tabular}{|c|c|c|c|c|}
\hline \multirow{2}{*}{} & \multicolumn{2}{|c|}{ Sample l } & \multicolumn{2}{c|}{ Sample 2 } \\
\cline { 2 - 5 } & from I $\mathrm{pp}$ & from I $_{\mathrm{pg}}$ & from I $_{\mathrm{pp}}$ & from I $\mathrm{pg}$ \\
\hline$\phi_{\mathrm{S}}$ & .2212 & .204 & .1531 & .139 \\
\hline $\mathrm{h}_{0}\left(\mathrm{~A}_{i}\right.$ & 452 & 479 & 385 & 420 \\
\hline $\mathrm{h}_{(\mathrm{A})}$ & .63 & 555 & 480 & 474 \\
\hline $\mathrm{u}_{\mathrm{yyyy}}$ & 803 & .863 & .802 & .886 \\
\hline$\gamma$ & 88.30 & 77.72 & 52.13 & 44.82 \\
\hline$\gamma_{0}$ & 133 & .019 & .07 & .007 \\
\hline$\chi^{2}$ & 13.82 & 5.447 & 2.115 & 3.524 \\
\hline
\end{tabular}




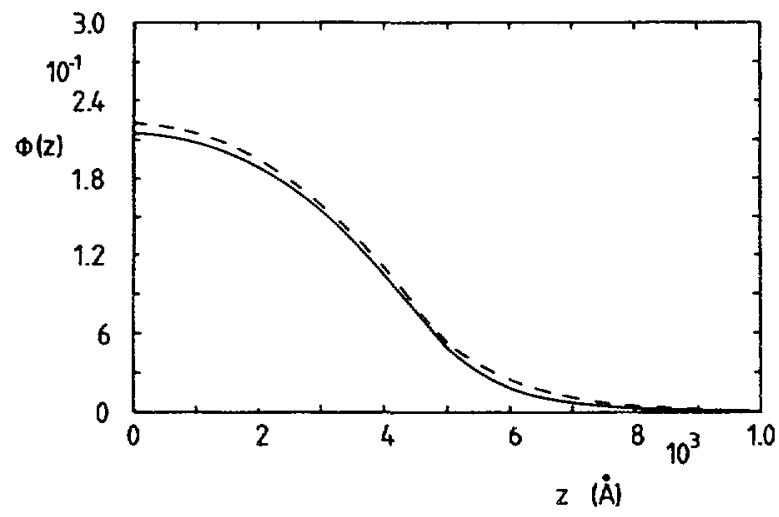

Fig. 6. - Comparison between the best density profiles, assuming a parabolic shape but with a background of the form of $\frac{\gamma_{0}}{q^{2}}$ (solid line) or of the form of $\gamma_{0}$ (dashed line). Sample 1 , experiment performed at contrast match.

It is possible to analyse the data without introducing this ad-hoc background, but allowing the density profile to take a surface effect into account explicitly. For this purpose, $\phi(z)$ is decomposed into two (or three) parts : far from the surface (central region), it follows the parabolic profile and close to the wall, it is assumed to have a different shape. The crossover distance is an adjustable parameter and again, we impose that $\phi(z)$ and its derivative remain continuous. In order to be consistent with the above discussion, we have restricted our choice to curves that can be characterized by 4 parameters. Three types of profile have been tested, allowing first to determine the sign of the surface effect (adsorption versus depletion), second to describe in more detail the shape of $\phi(z)$ in this proximal region and finally to evaluate the importance of this effect compared to the foot-like correction (since both modify the spectra approximately in the same $q$ range and in the same way).

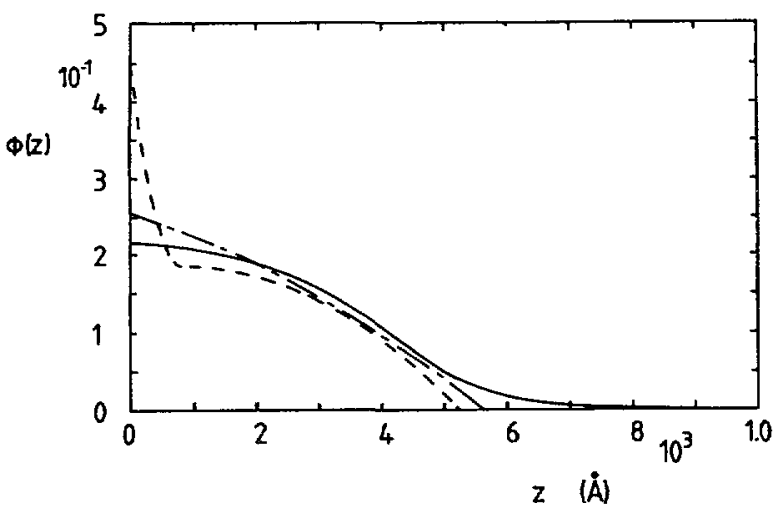

Fig. 7. - Test of the proximal region. Sample 1, experiment performed at contrast match. Best density profiles assuming a parabolic (dashed) or an exponential shape (dashed-dotted) in the proximal region. The central region is parabolic. No tail has been added. The solid curve corresponds to the best parabolic density profile as determined above (see text 2.4 and Fig. 5). 
Table VIII. - Investigation of the proximal region (of width $h_{0}$ ). The central region is assumed to be a parabola of extension $h$. No exponential foot has been added. Two forms for the proximal region have been tested : parabolic or exponential, corresponding to the 2 rows of this table. As before, $\phi_{s}$ is the value of $\phi(z)$ at $z=0 . \phi$ is the value of $\phi(z)$ at $z=h_{0}$. See figure 7 and text.

\begin{tabular}{|c|c|c|c|c|c|c|}
\hline & $\phi_{\mathrm{S}}$ & $\phi$ & $\gamma_{(\dot{\mathcal{A}})}$ & $h_{(\mathcal{A})}$ & $h_{0}$ & $\chi^{2}$ \\
\hline Parabola & .450 & .182 & 75.76 & 523 & 82 & 64.72 \\
\hline Exponential & .255 & .229 & 80.82 & 563 & 79 & 20.04 \\
\hline
\end{tabular}

In figure 7 , the dashed curve corresponds to the case of a proximal region described by a piece of a parabola. The link with the central region is imposed to be parallel to the $z$-axis (zero derivative). This kind of profile allows us to take into account, a priori, the effect of the surface whether it induces a depletion or an adsorption layer. The values of the different parameters are shown in table VIII. We can conclude from this first test that there is no depletion close to the wall but very likely an adsorption layer. This is not surprising since we have observed that the non-reactive polymer, put against the silica surface under the same conditions as the for the reactive chains, adsorb slightly onto this surface. (The amount of adsorbed poly(styrene) is more than three times smaller than the amount of grafted poly(styrene).) In figure 7, we have also reported the best profile corresponding to the case of an adsorption layer described by an exponential (dotted-dashed) and for comparison, the parabola determined in the previous part (solid curve) - cf. 2.4. It is clear, at least from the values of $\chi^{2}$, that the proximal region is much better described by an exponential than by a parabola. However, all these precise features can be considered as details of relatively little importance : the central region of the density profile is very robust. By comparison, the introduction of the tail far from the surface appears to be more significant.

It was tempting to keep the latter ingredient and simultaneously to try to take into account the adsorption directly in terms of $\phi(z)$. Since we have restricted our choice to only 4 parameter curves and since we impose that $\phi(z)$ and its derivative remain continuous, there is only one

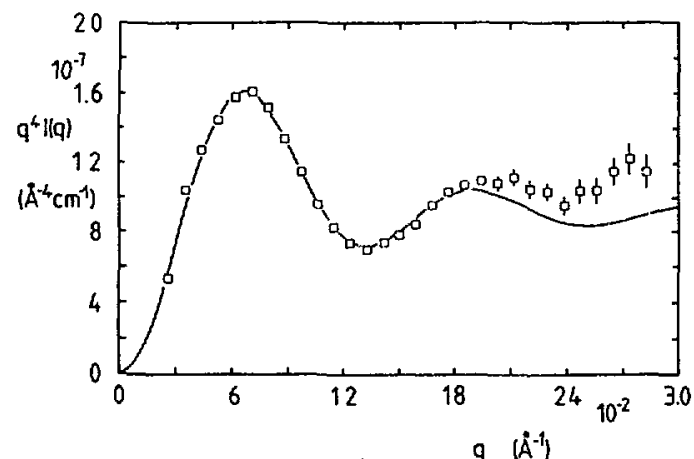

a)

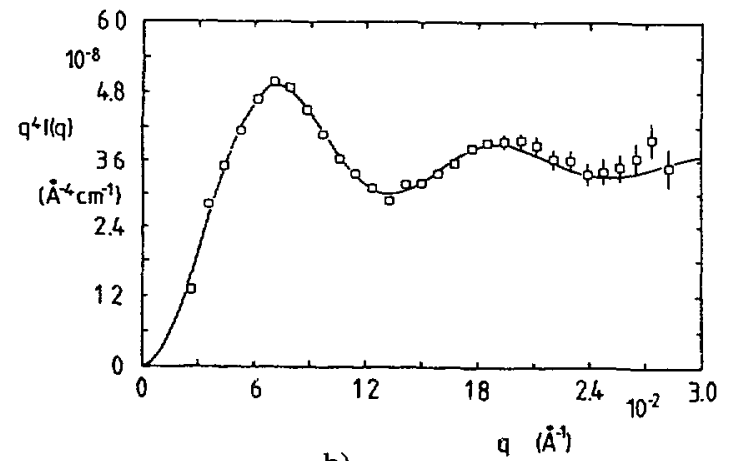

b)

Fig. 8. - Plot of $q^{4} I_{\mathrm{pp}}(q)$ versus $q$. a) sample 1 and b) sample 2 . The solid line corresponds to the best fit assuming a parabolic density profile with a non-zero tangent at $z=0$ and an exponential tail far from the surface. (The corresponding $\phi(z)$ are shown in Fig. 9a.) 
Table IX. - Same as for table III but instead of an ad-hoc background, we have introduced the parameter $\tau$ which accounts for the surface effect. $\tau$ is analogous to $\phi_{\mathrm{s}}$ and has no dimension. See note [15].

\begin{tabular}{|c|c|c|c|c|}
\hline \multirow{2}{*}{} & \multicolumn{2}{|c|}{ Sample 1 } & \multicolumn{2}{c|}{ Sample 2 } \\
\cline { 2 - 5 } & from I $_{\mathrm{pp}}$ & from I $_{\mathrm{pg}}$ & from I $_{\mathrm{pp}}$ & from I $_{\mathrm{pg}}$ \\
\hline$\phi_{s}$ & .2468 & .2225 & .1721 & .1515 \\
\hline $\mathrm{h}_{0}(\mathrm{~A})$ & 511 & 524 & 421 & 457 \\
\hline$h_{(\AA)}$ & 583 & 576 & 504 & 493 \\
\hline$u_{1}$ & .877 & .910 & .835 & .927 \\
\hline$\gamma$ & 81.81 & 77.02 & 51.06 & 45.15 \\
\hline$\tau$ & .086 & .126 & .071 & .091 \\
\hline$\chi^{2}$ & 3.984 & 1.374 & 2.391 & 2.342 \\
\hline
\end{tabular}

possibility for this purpose : it is to allow the slope of the tangent of the parabolic form to be non-zero at $z=0$. The results of this attempt are shown in figures 8 and 9 and the corresponding values of the parameters are displayed in table IX. If we compare these curves with those obtained in part 2.4. $\left(\phi_{s}\left(1-\left(\frac{z}{h}\right)^{2}\right)\right.$, exponential tail and background $\left.\frac{\gamma_{0}}{q^{2}}\right)$ - see figures $9 \mathrm{~b}$ and $9 \mathrm{c}$, we can notice that, on the whole, we obtain very close (and consistent) results. However, it also appears that the value of $\chi^{2}$ has decreased for the spectra obtained with the contrast variation method and increased for those obtained at contrast matching. We can also observe that the "fourth» parameter $\tau$ [15] which accounts for the surface effect is nearly the same for the contrast matching and the contrast variation experiment. (In part 2.4, $\gamma_{0}$ varied from more than one order of magnitude.) Therefore, we can conclude, concerning this discussion « background versus adsorption layer », that, probably, there is an adsorption layer close to the wall that has to be taken into account, but also some additional contribution to the scattering intensity at contrast matching, likely due to concentration fluctuations.

\section{Conclusion.}

On two samples, which differ only in their grafting density, we have tested different models describing the density profile of polymer brushes in good solvent. We have found that, among them, only the parabolic form agrees satisfactorily with the data. This parabola itself should be modified, far from the surface, with an exponential tail and close to the wall with an adsorption layer. The noise induced by concentration fluctuations is probably significant (in the $q$ range we have considered in this paper) only for the experiments done at contrast match. Finally, we point out that all this discussion has been possible because of the combination of well-defined samples and a sensitive technique. If one of these two partners were lacking, everything would break down. 


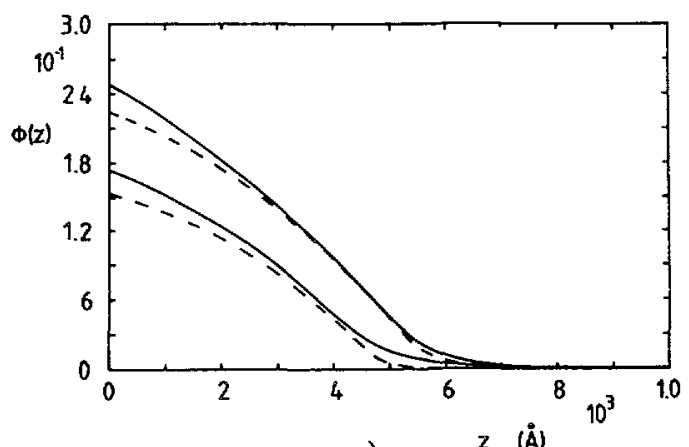

a)

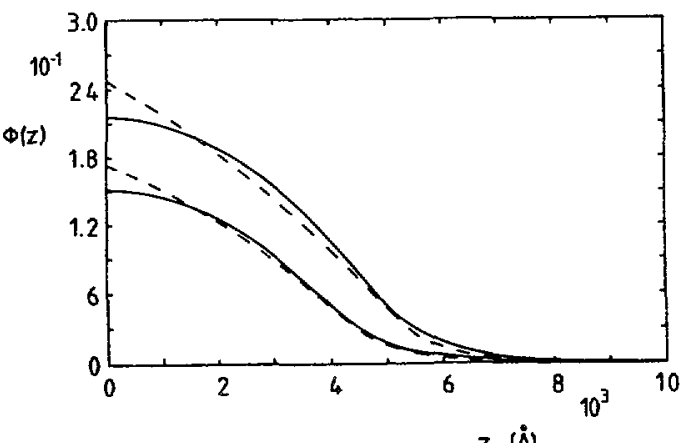

b)

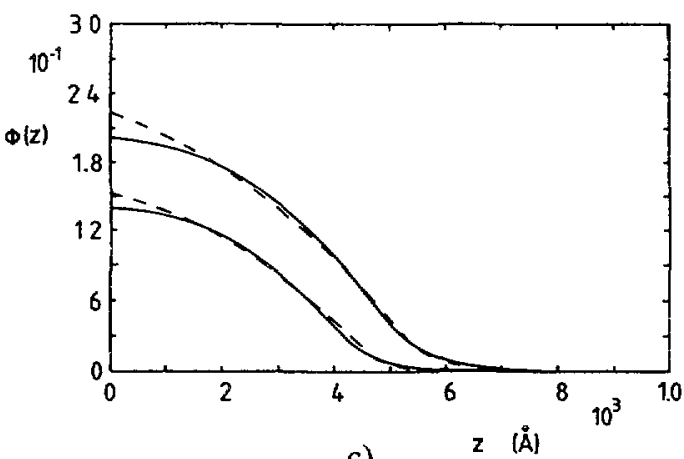

c)

Fig. 9. - a) Best density profiles assuming a parabolic shape with a non-zero tangent at $z=0$ and an exponential tail far from the surface. Solid curves : data obtained at contrast match. Dashed curves : data obtained with the contrast variation method. The two upper curves correspond to sample 1. b) Experiment performed at contrast match. Comparison between the best density profiles assuming either a zero tangent at $z=0$ and a background of the form of $\frac{\gamma_{0}}{q^{2}}$ (solid lines) or a non-zero tangent at $z=0$ and no background (dashed lines). The two upper curves correspond to sample 1. c) Same as for b, but now, the corresponding data have been obtained with the contrast variation method. 


\section{References}

[1] For a recent review, see : Halperin A., TiRrell M., Lodge T. P., Adv. Poly. Sci. 31 (1992) 100.

[2] For a review, see: Patel S., Tirrell M., Ann. Rev. Phys. Chem. 40 (1989).

[3] Alexander S., J. Phys. France 38 (1977) 983 ;

DE GENNES P. G., Macromolecules 13 (1980) 1069.

[4] Pryamitsyn V. A., Borisov O. V., Zhulina E. B., Birshtein T. M., Modern Problems of Physical Chemistry of Solutions (Donish and Leningrad University, 1987) p. 62 ;

Milner S. T., Witten T. A., Cates M. E., Europhys. Lett. 5 (1988) 413.

[5] Barnetr K., Cosgrove T., Vincent B., Burgess A., Crowley T., King T., Turner J., Tadros T., Polymer 22 (1981) 283 ;

Crowley T., Ph. D. Thesis (Oxford, 1981);

Cosgrove T., Heath T., Ryan K., Crowley T., Macromolecules 20 (1987) 2879.

[6] Auvray L., Auroy P., Neutron, X-Ray and Light Scattering, P. Lindner and Th. Zemb Eds (Elsevier Science Publishers B. V., 1991) p. 199.

[7] Cosgrove T., Heath T. G., Phipps J. S., Richardson R. M., Macromolecules 24 (1991) 94 ; Field J. B., Toprakcioglu C., Ball R. C., Stanley H. B., Dai l., Barford W., Penfold J., Smith G., Hamilton W., Macromolecules 25 (1992) 434 ;

Guiselin O., Lee L. T., Farnoux B., Lapp A., J. Chem. Phys. 95 (1991) 6 ;

KENT M. S., LEE L. T., RONDELEZ F., preprint ;

Satija S. K., Ankner J. F., Majkrzak C. F., Mansfield T., Beaucage G., Stein R. S., Iyengar D. R., MCCARTHY T. J., preprint.

[8] Auroy P., Mir Y., Auvray L., Phys. Rev. Lett. 69 (1992) 93.

[9] Auvray L., C.R. Acad. Sc. Paris, Ser. 302 (1986) 859 ; See also reference [6].

[10] This is also an argument in favour of uncorrect normalization for explaining the slight difference of the values of $\phi_{s}$ and $y$ when they are obtained under contrast matching condition or with the contrast variation method (see Sect. 2.2).

[11] Auroy P., Auvray L., LEger L., Phys. Rev. Lett. 66 (1991) 719.

[12] All this discussion about the meaning of the two corrections can be extended exactly in the same way to the linear and to the cubic shapes. The same conclusion would be drawn.

[13] WitTen T. A., Leibler L., Pincus P., Macromolecules 23 (1990) 824.

[14] This is a rough approximation. Indeed, it would be necessary to take into account some size distribution of the correlated volumes since the density profile is not a step. This would modify the Lorentzian behavior at small $q$. This second-order effect is neglected.

[15] $\tau$ is a.dimensionless parameter, analogous to $\phi_{s}, \tau>\phi_{s}$ corresponds to a depletion layer and $\tau<\phi_{\mathrm{s}}$ to an adsorption layer. The slope of the density profile at $z=0$ is $\frac{\tau-\phi_{\mathrm{s}}}{h}$ 